\title{
Thermoregulation-Independent Regulation of Sleep by Serotonin Revealed in Mice Defective in Serotonin Synthesis ${ }^{\mathrm{S}}$
}

\author{
Xian Zhang, ${ }^{1}$ Hongming Yan, ${ }^{1}$ Yanjia Luo, Zili Huang, and Yi Rao \\ School of Life Sciences, Beijing Normal University, Beijing, China (H.Y.); Peking-Tsinghua Center for Life Sciences, PKU- \\ IDG/McGovern Institute for Brain Research, Beijing Innovation Center for Genomics, Peking University School of Life Sciences, \\ Beijing, China (X.Z., H.Y., Y.R.); Department of Pharmacology, Fudan University School of Medicine, Shanghai, China (Y.L., Z.H.); \\ and Chinese Institute for Brain Research, Zhongguanchun Life Sciences Park, Beijing, China (Y.R.)
}

Received November 22, 2017; accepted April 3, 2018

\begin{abstract}
A role for 5-hydroxytryptamine (5-HT) or serotonin in sleep has been known for decades but was challenged by recent papers that concluded that the apparent sleep phenotype was secondary to defective thermoregulation. Those studies used mice lacking serotonergic neurons resulting from the loss of function mutations in the gene encoding the LIM homeobox transcription factor $1(L m \times 1 b)$. Here we show that, while $L m \times 1 b$ mutants failed to keep the physiologic body temperature, they exhibited more activities at the room and elevated temperatures.
\end{abstract}

More importantly, we used mice deficient in the gene encoding tryptophan hydroxylase 2 (Tph2), which could not synthesize 5-HT in the brain. Tph2 mutants were capable of thermoregulation and keeping physiologic body temperature when the environmental temperature was reduced and exhibited significantly more activities at both the room and elevated temperatures. Electroencephalographic (EEG) recording also showed decreased sleep in Tph2deficient mice. Our results indicate that $5-\mathrm{HT}$ is important for sleep regulation but not thermoregulation.

\section{Introduction}

The monoamine neurotransmitter 5-hydroxytryptamine (5-HT), also known as serotonin, is important in multiple physiologic processes. It is surprising that one of the earliest suggested roles for 5-HT, i.e., that in sleep regulation, remains controversial after decades of physiologic and pharmacological studies.

Early studies used $p$-chlorophenylalanine (pCPA), an inhibitor of tryptophan hydroxylase to deplete 5-HT from cats, rats, monkeys, and humans (reviewed in Jouvet, 1969, 1972). Reduction of central 5-HT levels after pCPA administration in cats (Koella et al., 1968) and rats (Mouret et al., 1968) were found to correlate with reduction of sleep. Damage of serotonergic neurons in the raphe nuclei of cats resulted in a decrease of sleep in a manner proportional to the size of the lesion (Jouvet et al., 1967; Jouvet, 1968).

We are grateful to the National Natural Science Foundation of China (Project 31421003 to Y.R.), the Beijing Innovation Center for Genomics, the Beijing Municipal Commission of Science and Technology (No. Z151100003915121) for grant support.

${ }^{1}$ X.Z. and H.Y. contributed equally to this work as first authors.

https://doi.org/10.1124/mol.117.111229.

S This article has supplemental material available at molpharm. aspetjournals.org.
5-Hydroxytryptophan (5-HTP) is a precursor of 5-HT capable of crossing the blood-brain barrier and can bypass the effect of pCPA to restore the cerebral 5-HT level. Injection of 5-HTP proportionally increased sleep and 5-HT levels in cats whose sleep was disrupted by pCPA (Mouret et al., 1967; Jouvet, 1968; Koella et al., 1968; Pujol et al., 1971). These studies led to the hypothesis that central 5-HT promotes sleep.

However, the discovery that administration of either 5-HT or 5-HTP in animals with normal 5-HT level sometimes did not induce physiologic sleep put the hypothesis in question (Jouvet, 1972). Large doses of 5-HTP increased electroencephalography (EEG) synchronization, a hallmark of neural activity during non-rapid eye movement (NREM) sleep, while suppressing rapid eye movement (REM) sleep in cats (Pujol et al., 1971). In monkeys, 5-HTP caused hyperactivity followed by an inactive period alternating between dozing and staring blankly (Macchitelli et al., 1966). Gain-of-function experiments suggested that 5 -HT was not simply promoting sleep or triggering sleep onset.

To further complicate the scenario, unit activities in raphe nuclei increased during waking, progressively decreased when animals transitioned from drowsiness to NREM sleep, and was almost silent during REM sleep (McGinty and Harper, 1976; Trulson and Jacobs, 1979; Lydic et al., 1987).

ABBREVIATIONS: 5-HT, 5-hydroxytryptamine; 5-HTP, 5-hydroxytryptophan; EEG, electroencephalographic; EMG, electromyography; Lmx1b, LIM homeobox transcription factor 1; NREM, non-rapid eye movement; pCPA, $p$-chlorophenylalanine; REM, rapid eye movement; Tph2, tryptophan hydroxylase 2; WT, wild type. 
Microdialysis measurement of extracellular 5-HT concentration from the dorsal raphe nucleus in cats showed that the 5-HT level was the highest during waking and progressively decreased from NREM to REM sleep (Portas and McCarley, 1994). However, some atypical raphe neurons showed higher activity during REM or NREM sleep or both in freely moving cats (Sakai and Crochet, 2001).

Genetic depletion of serotonergic neurons was achieved by ePet1-Cre driven knockout of $L m \times 1 b$ gene $\left(L m \times 1 b^{f / f / p}\right)$ (Zhao et al., 2006). $L m x 1 b^{f / f / p}$ mice exhibited increased wakefulness and decreased sleep (Buchanan and Richerson, 2010), but these results were attributed to the failure in maintaining body temperature, which caused the animals to exert hyperactivity to stay warm (Hodges et al., 2008; Buchanan and Richerson, 2010). This conclusion was not consistent with previous findings that suggested serotonergic neurons involved in thermoregulation were confined in the raphe magnus and the medullar nuclei (Berner et al., 1999; Tanaka et al., 2002; Nakamura et al., 2004), whereas the level of insomnia caused by lesion was proportional to the size of destruction, which suggested that 5-HT concentration, rather than specific nuclei, was important for sleep-wake regulation (Jouvet, 1969). A large proportion of raphe serotonergic neurons also release glutamate (Hioki et al., 2010; Liu et al., 2014; Sos et al., 2016), raising the possibility that distinct neurotransmitters are involved in divergent functions of raphe neurons.

We used Tph2 mutant mice, in which the raphe neurons are present but 5-HT could not be synthesized in the brain. We found that the $T p h 2^{-/-}$mice were not defective in thermoregulation at cold or normal ambient temperature. Sleep was decreased in these mutant mice, as shown by locomotive activities and by electroencephalography/electromyography (EEG/EMG) recordings.

\section{Materials and Methods}

Experimental Animals. All procedures and protocols were carried out under the instruction of the Institutional Animal Care and Use Committee at Peking University. Briefly, mice were kept under a 12:12-hour dark/light cycle at the ambient temperature of $25 \pm 1^{\circ} \mathrm{C}$. Experiments were carried out between 12 and 18 weeks of age. ePet1-Cre, $L m x 1 b^{f / f}$, and Tph2 mice were gifts from Dr. Zhoufeng Chen. Generation, breeding, and genotyping of $L m \times 1 b^{f / f / p}$ (Zhao et al., 2006) and Tph $2^{-1-}$ (Kim et al., 2014) mice have been described previously (Liu et al., 2011). Primers used for genotyping were AGGCTCCATCCATTCTTCTC and CCACAATAAGCAAGAGGCAC for $L m \times 1 b^{f / f}$; ATTTGCCTGCATTACCGGTCG and CAGCATTGCTGTCACTTGGTC for ePet1-Cre; and GGGCATCTCAGGACGTAGTAG, GGGCCTGCCGATAGTAACAC, and GCAGCCAGTAGACGTCTCTTAC for $T p h 2$. All strains were backcrossed with C57B/L for over eight generations in our laboratory before these experiments. For Tph2 mice, only males were used, whereas for $L m x 1 b^{f / f / p}$ mice, both males and females were used. Littermates with genotype $L m x 1 b^{f / f}$ or $T p h 2^{+/+}$ were used as controls for $L m x 1 b^{f / f / p}$ and $T p h 2^{-/-}$, respectively. All mice were housed individually during experiments. Numbers of male and female mice: Fig. 1 ( $n=7$ for $L m x 1 b^{f / f}$, 7 females, 0 males; $n=$ 4 for $L m x 1 b^{f / f / p}, 2$ females, 2 males; $n=6$ for $T p h 2^{+/+}, 5$ females, 1 male; $n=7$ for Tph $2^{-I-}, 7$ females, 0 males), Fig. $2(n=7$ for $L m \times 1 b^{f / f}, 7$ females, 0 males; $n=5$ for $L m x 1 b^{f / f / p}, 3$ females, 2 males; $n=7$ for $T p h 2^{+/+}, 6$ females, 1 male; $n=9$ for $T p h 2^{-/-}, 8$ females, 1 male), Fig. 3 (room temperature: $n=16$ for $L m x 1 b^{f / f}, 12$ females, 4 males; $n=12$ for $L m x 1 b^{f / f / p}, 6$ females, 6 males; $n=36$ for $T p h 2^{+/+}$, 29 females, 7 males; $n=33$ for $T p h 2^{-\prime-}, 27$ females, 6 males. $33^{\circ} \mathrm{C}$ : $n=15$ for $L m \times 1 b^{f / f}, 9$ females, 6 males; $n=13$ for $L m \times 1 b^{f / f / p}$,
7 females, 6 males; $n=30$ for $T p h 2^{+/+}, 21$ females, 9 males; $n=29$ for Tph $2^{-1-}, 20$ females, 9 males), Fig. 4 (all mice for EEG were male).

Core Body Temperature and Gross Motor Activity Measurement. Core body temperature and gross motor activity were measured by G2 E-Mitter implantable telemetry, acquired by ER4000 energizer/receiver, and analyzed by VitalView software (Starr Life Science, Oakmont, PA). Mice were anesthetized with tribromoethanol (250 mg/kg, i.p.), and electrodes were implanted intraperitoneally at the abdomen. Mice were individually housed and recovered for 1 week before recording. Data were recorded every 6 minutes during experiments. Mice were provided with free access to food and water during recording. For experiments at $4^{\circ} \mathrm{C}$, the $L m x 1 b$ mice were recorded in the cold room for at most 5 hours (and Tph2 mice for 9 hours) if they had not been removed because of a drop in body temperature.

Video Recording and Processing. Mice were recorded in their home cages for 3 days, and data acquired in the last 24 hours were used for analysis. For experiments at $33^{\circ} \mathrm{C}$, mice were kept in climate chamber (MGC-450HP-2; Yiheng Instruments, Kunshan, China) with 12:12-hour dark/light cycles and adequate food and water supply. Videos were captured at 1 frame per second (fps) and processed with lab-developed software. Briefly, the scale of each video was normalized according to standard grid, the region of interest was specified by hand, the threshold to distinguish mouse from background was selected from a binary representation of the original image, and the centroid of each mouse was automatically calculated and traced for activity. Only mice not moving for a consecutive 40 seconds were considered inactive according to previous studies (Pack et al., 2007), otherwise they were active or between activity bouts. Both the latter two states were counted as active.

EEG/EMG Recording and Processing. Mice were anesthetized with pentobarbital $(50 \mathrm{mg} / \mathrm{kg}$, i.p.) and chronically implanted with EEG and EMG electrodes for polysomnographic recordings according to previous studies (Qu et al., 2010). Two stainless steel screws (1 mm in diameter) were bilaterally inserted through the skull into the cortex $(1.0 \mathrm{~mm}$ anteriorly from bregma and $1.5 \mathrm{~mm}$ laterally to both sides from midline) according to the atlas (Paxinos and Franklin, 2008) to serve as EEG electrodes. Two Teflon-coated insulated stainless steel wires were placed bilaterally into trapezius muscles to serve as EMG electrodes. All electrodes were attached to a microconnector and fixed to the skull with dental cement.

The cable was connected through a slip ring to enable recording from free moving animals. Mice were individually housed for 10 days to recover and allowed to habituate to the recording cable for 3 to 4 days before polygraphic recording started. Each animal was recorded for 24 hours from the onset of dark phase at 7:00 PM.

EEG and EMG signals were amplified and filtered (EEG, $0.5-30 \mathrm{~Hz}$; EMG, 20-200 Hz), digitized at a sampling rate of $128 \mathrm{~Hz}$, and recorded by using SLEEPSIGN (Kissei Comtec, Nagano, Japan) as described earlier (Huang et al., 2005). The processed polygraphic signals were then automatically scored offline by 4-s epochs as wakefulness, REM, and NREM sleep by SLEEPSIGN according to standard criteria (Huang et al., 2005; Kohtoh et al., 2008). The defined sleep-wake states were finally examined manually and corrected, if necessary.

The Mann-Whitney test was carried out with GraphPad Prism [La Jolla, CA]).

\section{Results}

Neither $\operatorname{Lmx}_{1 b^{f f f} / p}$ nor $T_{p h 2^{-/}}$Mice Significantly Differ in Body Temperature from Wild-Type Mice. When serotonergic neurons were depleted with $L m \times 1 b$ floxp crossed to ePet1-cre $\left(\operatorname{Lm} x 1 b^{f / f / p}\right)$, mice were found to have higher body temperature in the dark phase than $L m \times 1 b$ floxp $\left(\operatorname{Lm} \times 1 b^{f / f}\right)$ controls during 12:12-hour dark/light cycles (Hodges et al., 2008). It was not possible to rule out whether this was attributable to heat generated from elevated activity. 
A

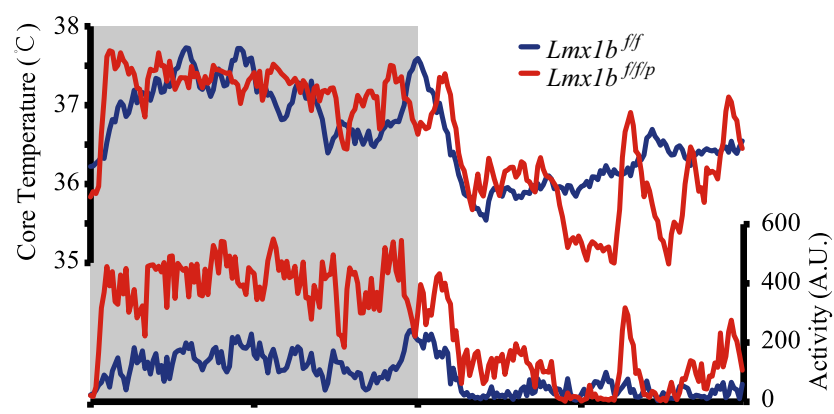

C

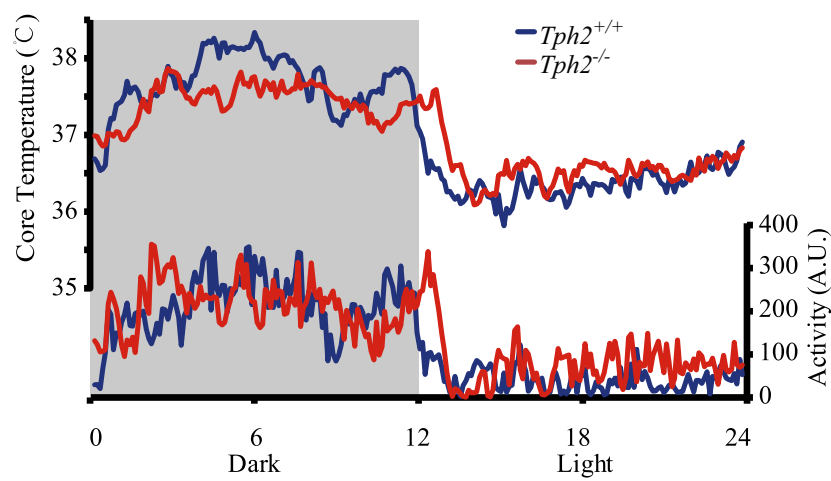

B

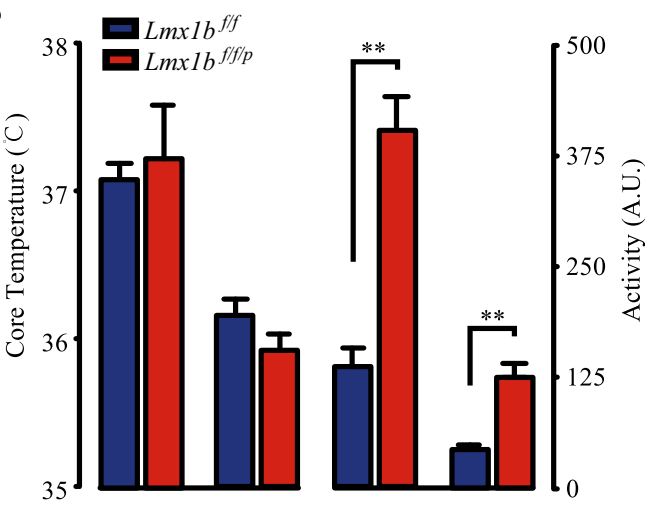

D

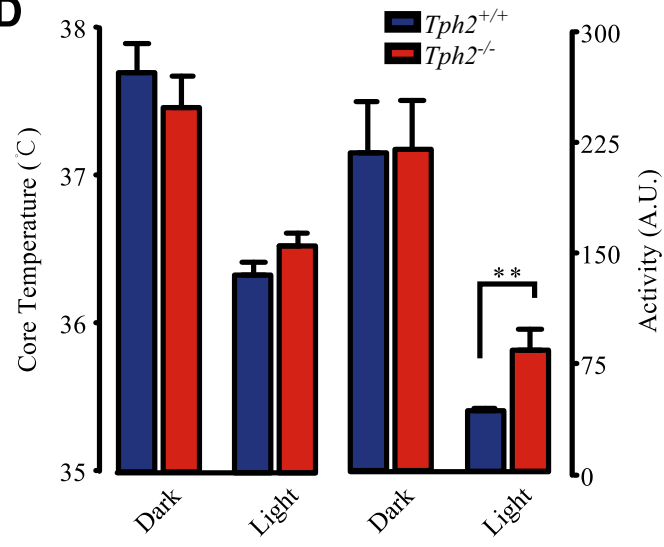

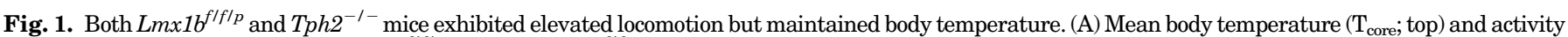

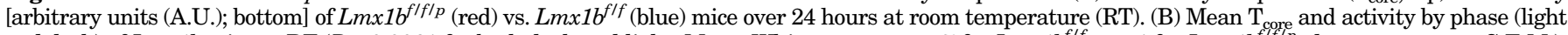

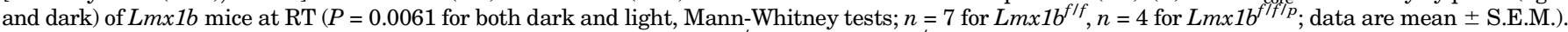

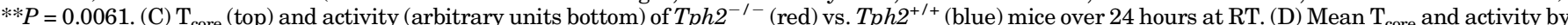


$* * P=0.0082$.

To examine whether Tph2-deficient mice were abnormal in thermoregulation, we measured the body temperature and activities of both $L m \times 1 b^{f / f / p}$ - and $T p h 2$-deficient mice by implantable emitter telemetry during a 24-hour light/dark cycle. All four groups of mice show fluctuations in body temperature, indicated as core temperature, within the 24-hour period: higher during the dark phase and lower during the light phase (Fig. 1). Different from the previous report (Hodges et al., 2008), we did not observe significant difference in body temperature between $L m \times 1 b^{\text {flf }}$ versus $L m x 1 b^{f / f / p}$ mice (Fig. 1, A and B), nor between Tph $2^{+/+}$and Tph $2^{-1-}$ mice (Fig. 1, C and D).

Movement of each mouse was measured as an indicator for activity simultaneously with the body temperature. All four groups of mice exhibited higher activity levels during the dark phase than those in the light phase (Fig. 1). $L m \times 1 b^{f / f / p}$ mice exhibited higher level of cumulative activity than $L m \times 1 b^{f / f}$ controls during both the dark and the light phases (Fig. 1, A and B), whereas the cumulative activity of $T p h 2^{-/-}$mice was only higher than the $T p h^{+/+}$ during the light phase (Fig. 1, C and D), when nocturnal animals spend most of the time sleeping. Elevated activities were also observed in previous studies (Hodges et al., 2008; Buchanan and Richerson, 2010) and were suggested to increase the body temperature. This explanation is not consistent with our results, because neither $L m \times 1 b^{f / f / p}$ nor $T p h 2^{-/-}$mice had elevated body temperature compared with their controls.

Neither $L m x 1 b^{f f f p}$ nor Tph2 $2^{-/-}$Mice Exhibited Excessive Activity during Cold Challenge Despite the Failure of $\operatorname{Lmx} 1 b^{f l f l p}$ to Maintain Body Temperature. To test the hypothesis that cold stress is the cause of elevated activity in the $L m x 1 b^{f / f / p}$-deficient mice, as suggested in the previous paper (Buchanan and Richerson, 2010), we measured the body temperature and locomotion of all four groups of mice at $4^{\circ} \mathrm{C}$ for 5 hours. $L m x 1 b^{f / f / p}$ mice indeed failed to maintain the body temperature, which dropped from $36.7 \pm 0.3^{\circ} \mathrm{C}$ to around $25^{\circ} \mathrm{C}$ (Fig. $2, \mathrm{~A}$ and C). By contrast, $T p h 2^{-I-}$ mice were able to maintain their body temperature within the same range as the $T p h 2^{+/+}$ mice (Fig. 2, D and F), suggesting that molecules other than 5-HT is involved in thermoregulation. Furthermore, we did not observe significantly elevated activity in either $L m \times 1 b^{f / f / p}$ or $T p h 2^{-l-}$ mice compared with their controls (Fig. 2, B, C, E, and F), which is against the hypothesis that excessive activities are responsible for counteracting body temperature loss.

Increased Activity in $\operatorname{Lmx} 1 b^{f l f l p}$ Mice was Not Diminished at Elevated Ambient Temperature. Since telemetry only offered a gross indicator of activity by recording the number of times that animals changed their locations, we also measured locomotion by video recording. Each group of 
A

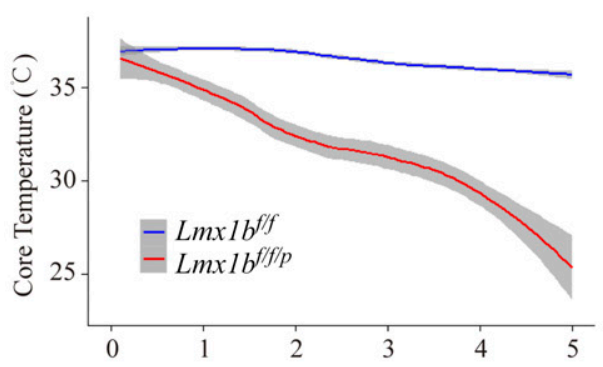

D



B



E





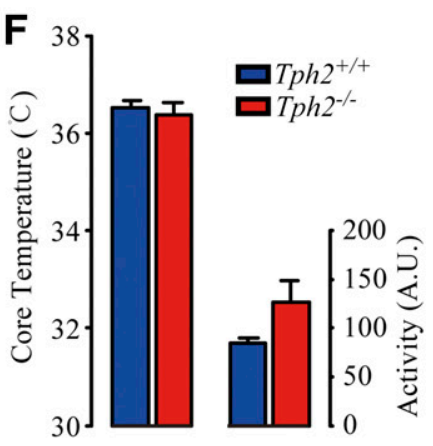

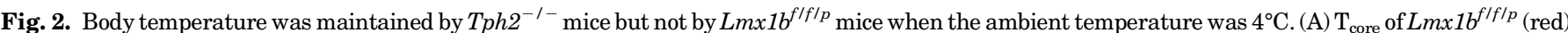

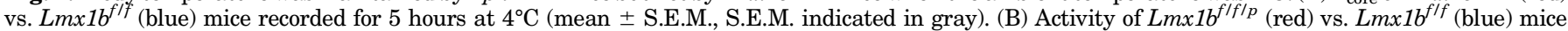

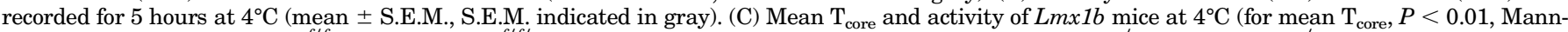

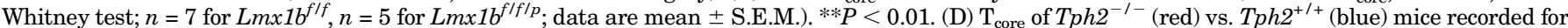


S.E.M. indicated in gray). (F) Mean $\mathrm{T}_{\text {core }}$ and activity of $\operatorname{Lmx} 1 b$ mice at $4^{\circ} \mathrm{C}\left(n=7\right.$ for $T p h 2^{+/+}, n=9$ for Tph2 $2^{-/-}$; data are mean \pm S.E.M.).

mice was recorded in home cages at either room temperature or $33^{\circ} \mathrm{C}$ for a $12: 12$-hour dark/light cycle. All mice retained normal 24-hour circadian cycles with more activities during the dark phase than the light phase. At room temperature, both $L m \times 1 b^{f / f / p}$ and $T p h 2^{-/-}$mice continuously displayed more activities than their controls. $L m \times 1 b^{f / f / p}$ mice moved a distance of $134.1 \pm 13.1 \mathrm{~m}$, whereas $L m x 1 b^{f / f}$ mice moved $30.9 \pm 5.0 \mathrm{~m}$ during the dark phase and $27.9 \pm 3.5 \mathrm{~m}$ versus $7.8 \pm 1.3 \mathrm{~m}$ during the light phase (Fig. 3, A and B), and also spent more time moving than $\operatorname{Lm} x 1 b^{f / f}$ (Fig. 3, C and D). Similarly, the distances traveled by $T p h 2^{-l-}$ versus $T p h 2^{+1+}$ mice (Fig. 3, E and $\mathrm{F}$ ) and duration of locomotion differed significantly (Fig. 3 , $\mathrm{G}$ and $\mathrm{H}$ ). Similar to a recent publication using adult-specific conditional knockout of Tph2 gene (Whitney et al., 2016), the active state of Tph $2^{-/-}$lasted for more than 1 hour after the light was on, whereas it was not observed in $L m x 1 b^{f / f / p}$ mice at $25^{\circ} \mathrm{C}$ (Fig. $3, \mathrm{~A}, \mathrm{C}, \mathrm{E}$, and G).

In contrast to the previous report of thermosensitive sleep recovery of $L m x 1 b^{f / f / p}$ mice at $33^{\circ} \mathrm{C}$ versus the room temperature (Buchanan and Richerson, 2010), we observed that $L m \times 1 b^{f / f / p}$ mice displayed higher activity levels during both the dark and light phases at $33^{\circ} \mathrm{C}$ (Fig. 3, I and J). Similar to our finding with telemetry (Fig. 1), $T p h 2^{-/-}$mice exhibited elevated activity only during the light phase at $33^{\circ} \mathrm{C}$ (Fig. 3, M-P). There was no significant differences in distance or duration during the dark phase at $33^{\circ} \mathrm{C}$ (Fig. 3, M-P). The diminished difference in locomotion between $T p h 2^{-/-}$and $T p h 2^{+/+}$mice during the dark phase was not due to decreased activity with Tph $2^{-1-}$, because the locomotion distances traveled at higher temperature increased with both $T p h 2^{+1+}$ and Tph $2^{-/-}$groups, but the increase with $T p h 2^{+/+}$mice was significantly larger (Supplemental Fig. S1). While $L m \times 1 b^{f / f / p}$ mice exhibited prolonged activity at $33^{\circ} \mathrm{C}$, $T p h 2^{-1-}$ mice displayed even higher level of activity after light on (Fig. 3, I, K, M, and O).

REM Sleep Was Decreased in Tph2 ${ }^{-1-}$ Mice. To directly verify whether 5-HT was involved in regulating sleep, we recorded the EEG/EMG to distinguish wakefulness, NREM, and REM sleep in $T p h 2^{+/+}$and $T p h 2^{-1-}$ mice for a 24-hour cycle at either room temperature or $33^{\circ} \mathrm{C}$. The EEG signal is high in frequency and low in voltage during wakefulness and REM sleep, and these features are reversed during NREM sleep with low frequency and high voltage. The EMG signal is high during wakefulness and low during both REM and NREM sleep, which, when combined with the EEG signal, unambiguously distinguishes the three states of sleep-wake cycle.

To our surprise, at room temperature, $T p h 2^{-l-}$ mice did not exhibit significantly more wakefulness than $T p h 2^{+1+}$ mice (Fig. 4, A-C). Neither did they differ in NREM sleep (Fig. 4, A-D). At room temperature, Tph $2^{-1-}$ mice showed significantly less REM than $T p h 2^{+/+}$during the light phase but not the dark phase (Fig. 4, A, B, and E).

At $33^{\circ} \mathrm{C}$, interestingly, the differences of wakefulness, NREM, and REM become larger between $T p h 2^{+/+}$and $T p h 2^{-1-}$ mice. During the dark phase, Tph $2^{-1-}$ mice stayed awake for longer periods (Fig. 4, F-H) and spent less time 




E

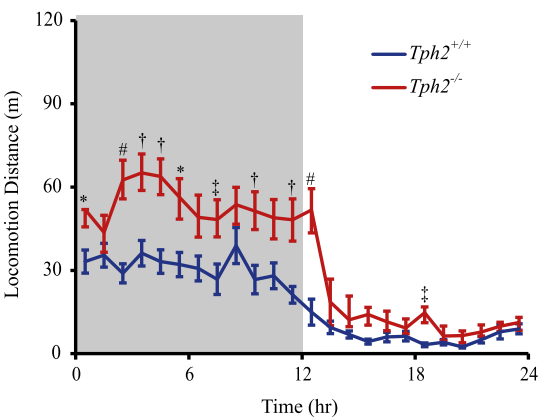

Locomotion Distance, $33^{\circ} \mathrm{C}$

I



M

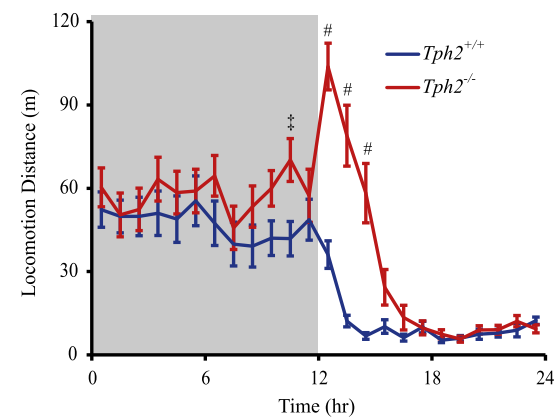

$\mathbf{F}$

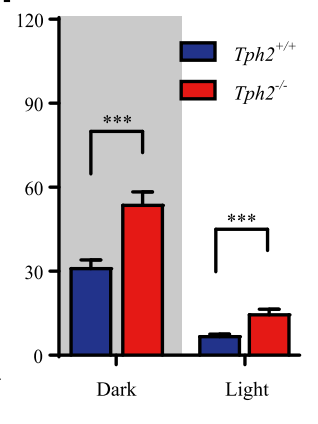

J

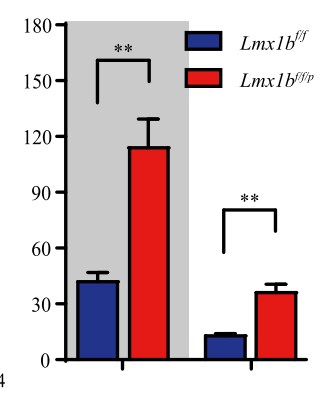

N

B
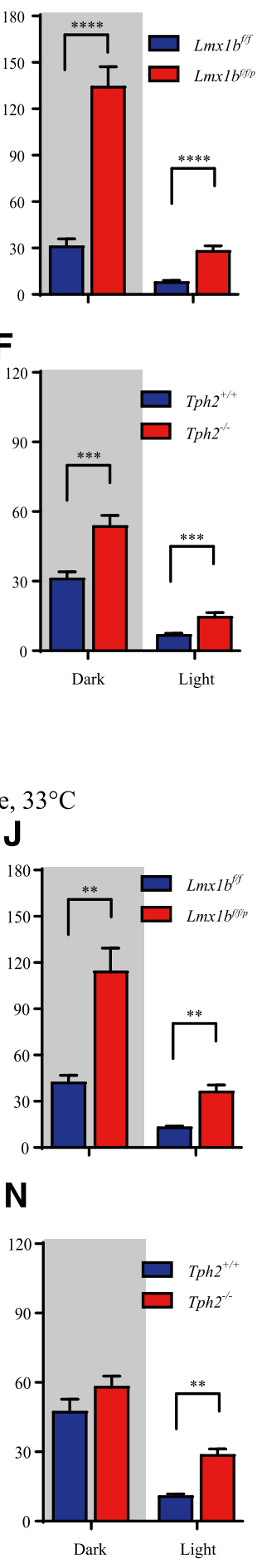

Activity Time, RT

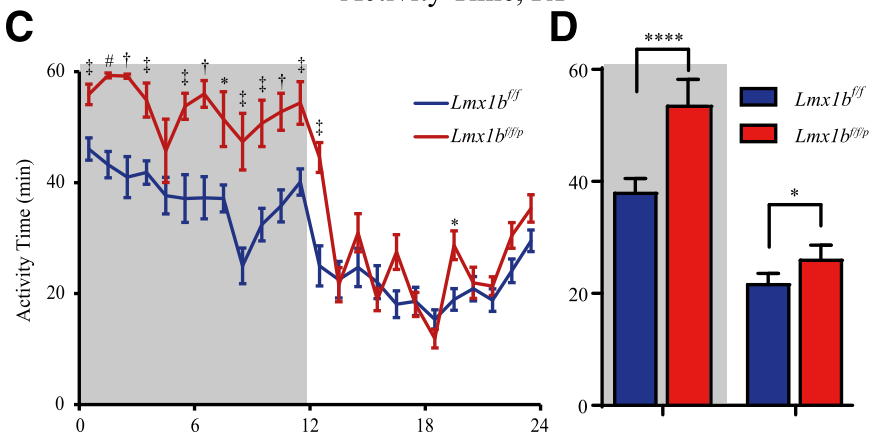

G
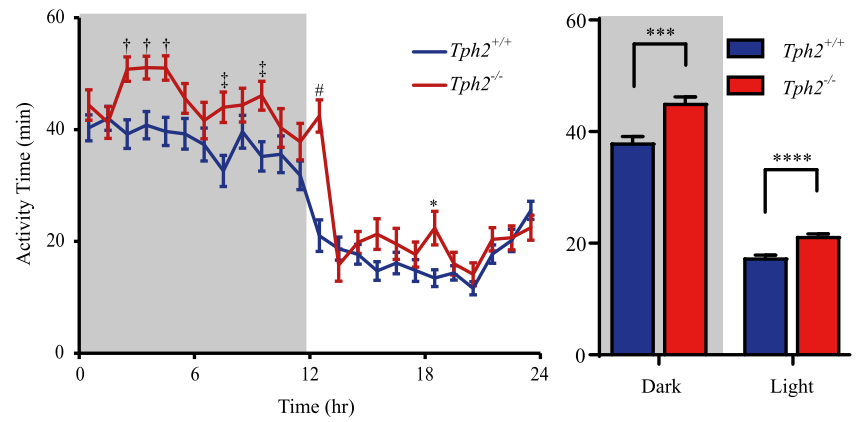

Activity Time, $33^{\circ} \mathrm{C}$

K

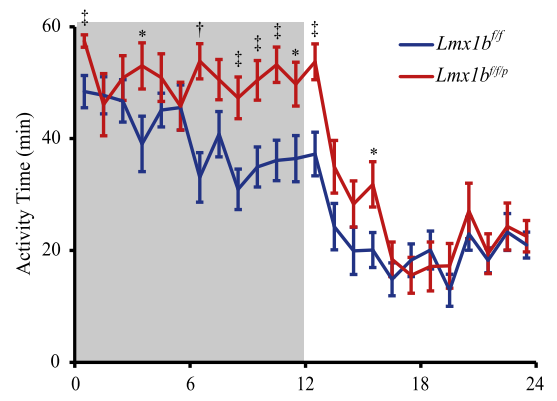

0

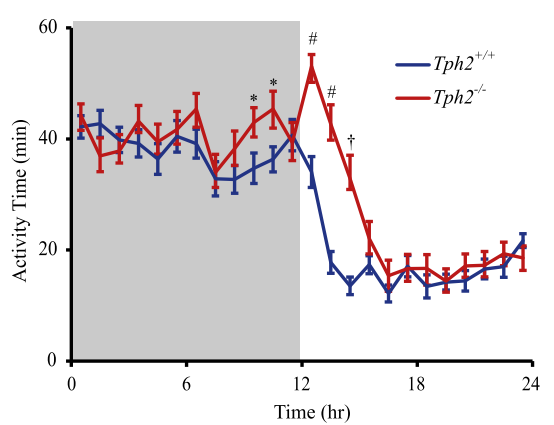

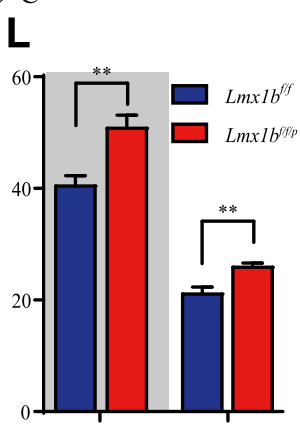

P

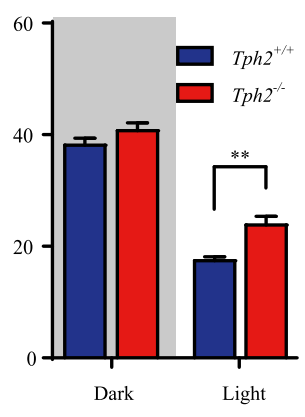

Fig. 3. Both $L m x 1 b^{f / f / p}$ and $T p h 2$ mice exhibited elevated activity at room temperature (RT) and $33^{\circ} \mathrm{C}$. (A and B) Mean locomotion distance of $L m x 1 b^{f / f / p}$ (red) vs. $L m x 1 f^{f / f}$ (blue) mice at RT $(P<0.0001$ for both dark and light phase in (B), Mann-Whitney tests; data are mean \pm S.E.M.). (C and D) Mean active time of $L m x 1 b^{f / f / p}$ (red) vs. $L m x 1 b^{f / f}$ (blue) mice at RT $(P<0.0001$ for dark phase and $P=0.0112$ for light phase in (D), Mann-Whitney tests; data are mean \pm S.E.M.; $n=16$ for $L m x 1 b^{f / f}, n=12$ for $L m x 1 b^{f / f / p}$ ). (E and F) Mean locomotion distance of $T p h 2^{-/-}$(red) vs. Tph $2^{+/+}$(blue) mice at RT $(P=0.0002$ for dark and $P=0.0006$



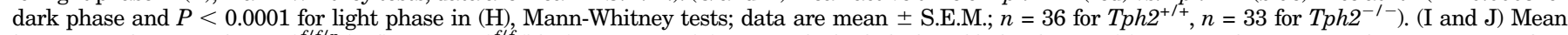
locomotion distance of $L m x 1 b^{f / f / p}$ (red) vs. $L m x 1 b^{f / f}$ (blue) mice at $33^{\circ} \mathrm{C}(P<0.01$ for both dark and light phase in (J), Mann-Whitney tests; data are mean \pm S.E. M.). (K and L) Mean active time of $L m x 1 b^{f / f / p}$ (red) vs. $L m x 1 b^{f / f}$ (blue) mice at $33^{\circ} \mathrm{C}[P<0.01$ for both dark and light phase in (L), Mann-Whitney tests; data are mean \pm S.E.M.; $n=15$ for $L m x 1 b^{f / f}, n=13$ for $\left.L m x 1 b^{f / f / p}\right]$. (M and N) Mean locomotion distance of $T p h 2^{-/-}$(red) vs. $T p h 2^{+/+}$(blue) mice at $33^{\circ} \mathrm{C}[P<0.01$ for the light phase in (N), Mann-Whitney tests; data are mean \pm S.E.M.]. (O and P) Mean active time of Tph $2^{-\prime-}$ (red) vs. $T p h 2^{+/+}\left(\right.$blue) mice at $33^{\circ} \mathrm{C}[P<0.01$ for the light phase in (P), Mann-Whitney tests; data are mean \pm S.E.M.; $n=30$ for $T p h 2^{+/+}, n=29$ for $T p h 2^{-}{ }^{-}$]. For (A, C, E, G, I, K, M, and O), ${ }^{*} P<0.05$; $* * P<0.01$; $* * *$ in F, $P=0.0002$ for first column, $P=0.0006$ for second column, in $\mathrm{H}, P=0.0005$; $* * * *$ in $\mathrm{D}, P<0.0001 ; \ddagger P<0.01 ; \dagger P<0.001$; \#P<0.0001; Mann-Whitney tests. 
$24 \mathrm{C}$

A



B

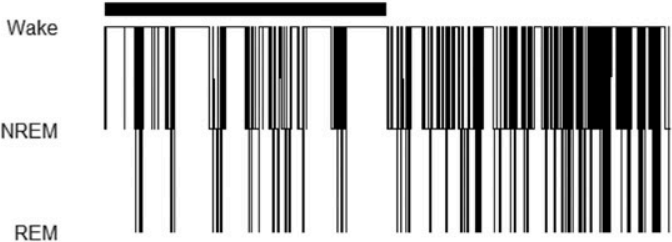

$33 \mathrm{C}$

$\mathbf{F}$

G

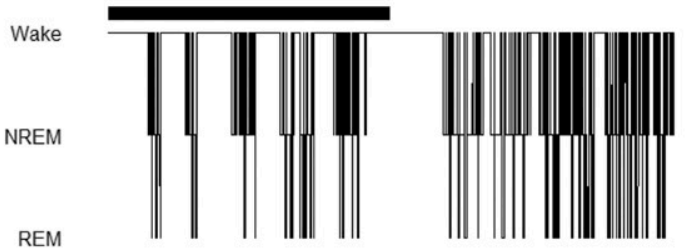

C

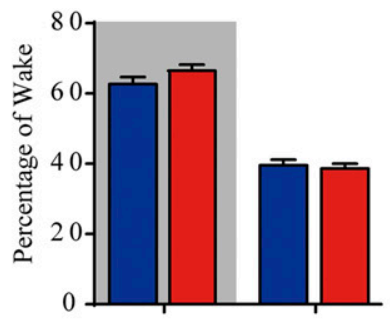

D

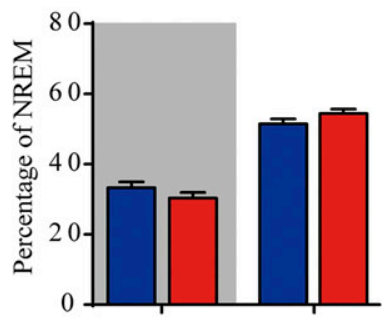

E

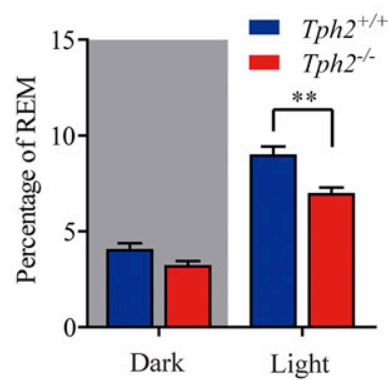

H

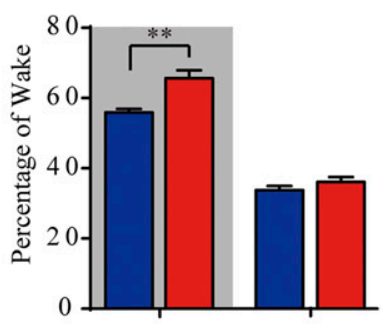

I

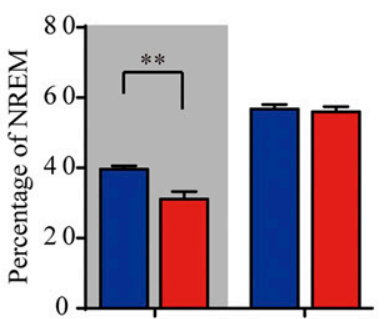

J

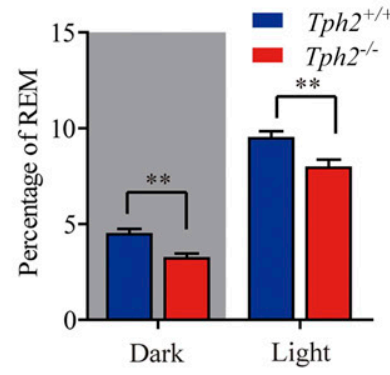

Fig. 4. Analysis of EEG/EMG patterns reveals that $T p h 2^{-1-}$ mice exhibited less sleep at both RT and $33^{\circ} \mathrm{C}$. (A and B) Examples of time spent on wake, NREM, and REM by $T p h 2^{++_{+}}(\mathrm{A})$ and $T p h 2^{-{ }^{-}}$(B) over 24 hours at RT. (C-E) Mean percentage of wake (C), NREM (D), and REM (E) by phase (light and dark) at RT $[P<0.01$ for light phase in (E), Mann-Whitney test; data are mean \pm S.E.M.; $n=20$ for $T p h 2^{+/+}$, $n=22$ for $T p h 2^{-1-}$. (F and G) Examples of time spent on wake, NREM, and REM by $T p h 2^{+/+}(\mathrm{F})$ and $T p h 2^{-1-}(\mathrm{G})$ over 24 hours at $33^{\circ} \mathrm{C}$. (H-J) Mean percentage of wake $(\mathrm{H}), \operatorname{NREM}(\mathrm{I})$, and REM $(\mathrm{J})$ by phase (light and dark) at $33^{\circ} \mathrm{C}[P<0.01$ for dark phase in (H), $P<0.01$ for dark phase in (I), $P<$ 0.01 for both dark and light phase in $(J)$; Mann-Whitney test; data are mean \pm S.E. M.; $n=20$ for $T p h 2^{+/+}, n=21$ for $T p h 2^{-/-}$]. $* * P<0.01$. in both NREM (Fig. 4, F, G, and I) and REM sleep (Fig. 4, F, G, and J). During the light phase, Tph $2^{-/-}$mice also had less REM sleep than the Tph $2^{+/+}$(Fig. 4, F, G, and J). We did not observe a significant difference in the amount of wakefulness (Fig. 4, F-H) or NREM sleep (Fig. 4, F, G, and I) during the light phase at $33^{\circ} \mathrm{C}$. These results indicate that the sleep phenotype of $T p h 2^{-1-}$ mice is present at $33^{\circ} \mathrm{C}$. 


\section{Discussion}

5-HT and central serotonergic neurons in the raphe nuclei have been implicated in multiple physiologic processes, which complicated the endeavor to distinguish one role from another and also made it confusing whether a role was played by 5 -HT or by serotonergic neurons but with other transmitters. The function of 5-HT in sleep-wake regulation has remained under debate for many years, with recent papers concluding that the apparent sleep phenotype in mice lacking serotonergic neurons was explained by the primary role of serotonergic neurons in thermoregulation (Buchanan and Richerson, 2010). Here we provide multiple pieces of evidence indicating that the role of 5-HT in sleep is not attributable to its role in thermoregulation.

Mice depleted of serotonergic neurons were found to be defective in body temperature control as they were not able to keep normal temperature during coldness challenge (Hodges et al., 2008). However, a paper with a $T p h 2^{-/-}$strain, which deleted the coding part of Tph2 gene exon 1 and 2, did not report body temperature changes in $T p h 2$-deficient mice (Alenina et al., 2009). We used two types of mouse mutants to study the roles of serotonergic neurons and 5-HT in sleep regulation. One of them, $L m \times 1 b^{f / f / p}$ crossed with ePet1-cre, is the same as has been studied previously (Buchanan and Richerson, 2010). Mice lacking serotonergic neurons showed higher body temperature during the dark phase and were unable to maintain normal body temperature when challenged by coldness at $4^{\circ} \mathrm{C}$. We also found that the temperature of $L m \times 1 b^{f / f / p}$ mice dropped dramatically at $4^{\circ} \mathrm{C}$ within 5 hours (Fig. 2). Since hindbrain ePet1-positive neurons also contain other neurotransmitters such as glutamate and neuropeptides such as substance $\mathrm{P}$, we used $T p h 2^{-1-}$ mice to study the role of 5-HT (Zhao et al., 2006). We found that the body temperature of $T p h 2^{-1-}$ mice did not differ from that of wildtype (WT) mice and remained at the normal range at $4^{\circ} \mathrm{C}$. Another study found that, although the body temperature of

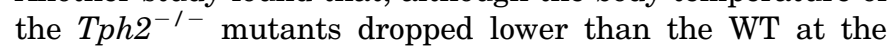
beginning of cold challenge, it recovered slowly to the same level as the WT at the end of the 4th hour (Alenina et al., 2009), supporting that mice lacking 5 -HT were not defective in thermoregulation.

Previous work with $L m x 1 b^{f / f / p}$ mice demonstrated that increased wakefulness and decreased sleep with the $L m \times 1 b^{f / f / p}$ disappeared as the ambient temperature increased from $24^{\circ} \mathrm{C}$ to $33^{\circ} \mathrm{C}$, leading to the conclusion that elevated activity and reduced sleep was due to deregulation of body temperature and perception of $24^{\circ} \mathrm{C}$ as cold stress. However, our results do not support this conclusion. First of all, another study from the same group demonstrated that $L m x 1 b^{f / f / p}$ mice preferred ambient temperature of $30.6^{\circ} \mathrm{C}$, which was similar to the $L m x 1 b^{f / f}$ (Hodges et al., 2008), suggesting that temperature perception in $L m \times 1 b^{f / f / p}$ mice was not different from the WT. Secondly, despite decreased body temperature at $4^{\circ} \mathrm{C}$, no elevated activity of $L m \times 1 b^{f / f / p}$ mice was observed by us during cold challenge (Fig. 2), raising the possibility that activity was not increased in these mice to generate heat. Moreover, $L m x 1 b^{f / f / p}$ mice exhibited higher level of locomotion activity at both room temperature and $33^{\circ} \mathrm{C}$. Similar to a recent publication with the $T p h 2$ gene conditionally deleted in the raphe nuclei of adult mice (Whitney et al., 2016), the $T p h 2^{-/-}$mice exhibited higher activity level in locomotion and activity time during both the dark and light phases at the room temperature, whereas elevation was only observed during the light phase at $33^{\circ} \mathrm{C}$ (Fig. 3). Activity was increased in $T p h 2^{-l-}$ mice as well as $L m \times 1 b^{f / f / p}$ at both the normal and warm ambient temperatures, suggesting a direct role of 5-HT in sleep-wake regulation. These results demonstrated that absence of 5-HT did not dramatically disrupt body temperature control. Therefore, the elevated activity in $T p h 2^{-/-}$mice could not be attributed to hypothermia. Thirdly, the EEG/EMG measurements further support our point. Compared with $T p h 2^{+/+}$mice, REM duration was reduced in $T p h 2^{-1-}$ mice at both room temperature and $33^{\circ} \mathrm{C}$ (Fig. 4). The changes in wakefulness and NREM sleep duration are less clear. We only observed marginal difference between $T p h 2^{-1-}$ and $T p h 2^{+1+}$ mice at room temperature. It was not significant until the ambient temperature was $33^{\circ} \mathrm{C}$ and only during the dark phase. The different tendency of changes from room temperature to $33^{\circ} \mathrm{C}$ between activity and sleep/wake duration may indicate an increase in passive wakefulness with $T p h 2^{-1-}$ and increased activity with $T p h 2^{+/+}$at warm temperatures.

Serotonergic receptors have been studied for their roles in sleep regulation (reviewed in Monti, 2011). For example, 5$\mathrm{HT}_{1 \mathrm{~A}}$ receptor is thought to act as a presynaptic self- inhibitory receptor, and REM sleep was increased in mouse mutants lacking $5-\mathrm{HT}_{1 \mathrm{~A}} \mathrm{R}$, which was opposite to the effect of 5-HT depletion (Boutrel et al., 2002). Consistently, Inhibition of $5-\mathrm{HT}_{1 \mathrm{~A}}$ receptors by antagonist WAY100635 increased REM sleep, whereas the agonist 8-hydroxy-2-(di- $n$-propylamino)tetralin (8-OH-DPAT) decreased REM sleep in both mice and cats (Portas et al., 1996; Boutrel et al., 2002). REM was also increased in mice lacking $5-\mathrm{HT}_{1 \mathrm{~B}} \mathrm{R}$ (Boutrel et al., 1999), which is thought to act as an inhibitory receptor, too. NREM sleep was decreased, wakefulness was increased, but REM sleep was not affected in $5-\mathrm{HT}_{2 \mathrm{~A}} \mathrm{R}$ mutant mice (Popa et al., 2005). Wakefulness was increased, NREM decreased, but REM was unaffected in mutants lacking the $5-\mathrm{HT}_{2 \mathrm{C}}$ receptor (Frank et al., 2002), which is similar to the sleep phenotype of mice lacking $5-\mathrm{HT}_{2 \mathrm{~A}} \mathrm{R}$. $\mathrm{REM}$ was decreased in mice lacking the $5-\mathrm{HT}_{7}$ receptor (Hedlund et al., 2005). In the future, it will be ideal to study mutants of 5-HT receptors of identical genetic background and with identical methods under the same conditions.

In summary, we found that dysfunction in thermoregulation could not explain the elevated wakefulness and decreased sleep level in animals lacking 5-HT, in that: 1) animals deficient in 5-HT synthesis were able to maintain body temperature in cold environment, 2) animals depleted of serotonergic neurons preferred the same ambient temperature as the WT mice, 3) animals lacking serotonergic neurons exhibited more activity at an elevated ambient temperature, and 4) animals deficient in 5-HT synthesis woke more and slept less even at an elevated ambient temperature. Thus, our study supports a role of 5-HT in sleep-wake regulation independent of thermoregulation.

\section{Acknowledgments}

The authors are grateful to Dr. Zhoufeng Chen for sharing the $T p h 2$ mice and to Yunxia Si and Xiangyun Yue for animal husbandry.

\section{Authorship Contributions}

Participated in research design: Zhang, Yan, Huang, Rao. 
Conducted experiments: Zhang, Yan, Luo.

Performed data analysis: Zhang, Yan, Luo.

Wrote or contributed to the writing of the manuscript: Zhang, Yan, Rao.

\section{References}

Alenina N, Kikic D, Todiras M, Mosienko V, Qadri F, Plehm R, Boyé P, Vilianovitch L, Sohr R, Tenner K, et al. (2009) Growth retardation and altered autonomic control in mice lacking brain serotonin. Proc Natl Acad Sci USA 106:10332-10337.

Berner NJ, Grahn DA, and Heller HC (1999) 8-OH-DPAT-sensitive neurons in the nucleus raphe magnus modulate thermoregulatory output in rats. Brain Res 831:155-164.

Boutrel B, Franc B, Hen R, Hamon M, and Adrien J (1999) Key role of 5-HT1B receptors in the regulation of paradoxical sleep as evidenced in 5-HT1B knock-out mice. J Neurosci 19:3204-3212.

Boutrel B, Monaca C, Hen R, Hamon M, and Adrien J (2002) Involvement of 5-HT1A receptors in homeostatic and stress-induced adaptive regulations of paradoxical sleep: studies in 5-HT1A knock-out mice. J Neurosci 22:4686-4692.

Buchanan GF and Richerson GB (2010) Central serotonin neurons are required for arousal to CO2. Proc Natl Acad Sci USA 107:16354-16359.

Frank MG, Stryker MP, and Tecott LH (2002) Sleep and sleep homeostasis in mice lacking the 5-HT2c receptor. Neuropsychopharmacology 27:869-873.

Hedlund PB, Huitron-Resendiz S, Henriksen SJ, and Sutcliffe JG (2005) 5-HT7 receptor inhibition and inactivation induce antidepressantlike behavior and sleep pattern. Biol Psychiatry 58:831-837.

Hioki H, Nakamura H, Ma YF, Konno M, Hayakawa T, Nakamura KC, Fujiyama F, and Kaneko T (2010) Vesicular glutamate transporter 3-expressing nonserotonergic projection neurons constitute a subregion in the rat midbrain raphe nuclei. J Comp Neurol 518:668-686.

Hodges MR, Tattersall GJ, Harris MB, McEvoy SD, Richerson DN, Deneris ES, Johnson RL, Chen Z-F, and Richerson GB (2008) Defects in breathing and thermoregulation in mice with near-complete absence of central serotonin neurons. $J$ Neurosci 28:2495-2505.

Huang Z-L, Qu W-M, Eguchi N, Chen J-F, Schwarzschild MA, Fredholm BB, Urade $\mathrm{Y}$, and Hayaishi $\mathrm{O}$ (2005) Adenosine A2A, but not A1, receptors mediate the arousal effect of caffeine. Nat Neurosci 8:858-859.

Jouvet M (1968) Insomnia and decrease of cerebral 5-hydroxytryptamine after destruction of the raphe system in the cat. Adv Pharmacol 6 (Pt B):265-279.

Jouvet M (1969) Biogenic amines and the states of sleep. Science 163:32-41.

Jouvet M (1972) The role of monoamines and acetylcholine-containing neurons in the regulation of the sleep-waking cycle, in Neurophysiology and Neurochemistry of Sleep and Wakefulness pp. 166-307, Springer, Berlin.

Jouvet M, Bobillier P, Pujol JF, and Renault J (1967) Suppression of sleep and decrease of cerebral serotonin caused by lesion of the raphe system in the cat. $C R$ Acad Sci Hebd Seances Acad Sci D 264:360-362.

Kim JY, Kim A, Zhao ZQ, Liu XY, and Chen ZF (2014) Postnatal maintenance of the 5 -Ht1a-Pet1 autoregulatory loop by serotonin in the raphe nuclei of the brainstem. Mol Brain 7:48.

Koella WP, Feldstein A, and Czicman JS (1968) The effect of para-chlorophenylalanine on the sleep of cats. Electroencephalogr Clin Neurophysiol 25:481-490.

Kohtoh S, Taguchi Y, Matsumoto N, Wada M, Huang ZL, and Urade Y (2008) Algorithm for sleep scoring in experimental animals based on fast Fourier transform power spectrum analysis of the electroencephalogram. Sleep Biol Rhythms 6:163-171.

Liu Y, Jiang Y, Si Y, Kim J-Y, Chen Z-F, and Rao Y (2011) Molecular regulation of sexual preference revealed by genetic studies of 5-HT in the brains of male mice. Nature 472:95-99.

Liu Z, Zhou J, Li Y, Hu F, Lu Y, Ma M, Feng Q, Zhang JE, Wang D, Zeng J, et al. (2014) Dorsal raphe neurons signal reward through 5-HT and glutamate. Neuron 81:1360-1374.

Lydic R, McCarley RW, and Hobson JA (1987) Serotonin neurons and sleep. I. Long term recordings of dorsal raphe discharge frequency and PGO waves. Arch Ital Biol 125:317-343.
Macchitelli FJ, Fischetti D, and Montanarelli N, Jr (1966) Changes in behavior and electrocortical activity in the monkey following administration of 5-hydroxytryptophan (5-HTP). Psychopharmacology (Berl) 9:447-456.

McGinty DJ and Harper RM (1976) Dorsal raphe neurons: depression of firing during sleep in cats. Brain Res 101:569-575.

Monti JM (2011) Serotonin control of sleep-wake behavior. Sleep Med Rev 15: $269-281$.

Mouret J, Bobillier P, and Jouvet M (1967) Effect of parachlorophenylalanine on sleep in rats. C R Seances Soc Biol Fil 161:1600-1603.

Mouret J, Bobillier P, and Jouvet M (1968) Insomnia following parachlorophenylalanine in the rat. Eur $J$ Pharmacol 5:17-22.

Nakamura K, Matsumura K, Hübschle T, Nakamura Y, Hioki H, Fujiyama F, Boldogköi Z, König M, Thiel H-J, Gerstberger R, et al. (2004) Identification of sympathetic premotor neurons in medullary raphe regions mediating fever and other thermoregulatory functions. $J$ Neurosci 24:5370-5380.

Pack AI, Galante RJ, Maislin G, Cater J, Metaxas D, Lu S, Zhang L, Von Smith R, Kay T, Lian J, et al. (2007) Novel method for high-throughput phenotyping of sleep in mice. Physiol Genomics 28:232-238.

Paxinos G and Franklin KBJ (2008) The Mouse Brain in Stereotaxic Coordinates, Compact, The Coronal Plates and Diagrams, 3rd ed, Elsevier Academic Press, San Diego, CA.

Popa D, Léna C, Fabre V, Prenat C, Gingrich J, Escourrou P, Hamon M, and Adrien J (2005) Contribution of 5-HT2 receptor subtypes to sleep-wakefulness and respiratory control, and functional adaptations in knock-out mice lacking 5-HT2A receptors. J Neurosci 25:11231-11238.

Portas CM and McCarley RW (1994) Behavioral state-related changes of extracellular serotonin concentration in the dorsal raphe nucleus: a microdialysis study in the freely moving cat. Brain Res 648:306-312.

Portas CM, Thakkar M, Rainnie D, and McCarley RW (1996) Microdialysis perfusion of 8-hydroxy-2-(di-n-propylamino)tetralin (8-OH-DPAT) in the dorsal raphe nucleus decreases serotonin release and increases rapid eye movement sleep in the freely moving cat. J Neurosci 16:2820-2828.

Pujol J-F, Buguet A, Froment J-L, Jones B, and Jouvet M (1971) The central metabolism of serotonin in the cat during insomnia. A neurophysiological and biochemical study after administration of P-chlorophenylalanine or destruction of the Raphé system. Brain Res 29:195-212.

Qu W-M, Xu X-H, Yan M-M, Wang Y-Q, Urade Y, and Huang Z-L (2010) Essential role of dopamine D2 receptor in the maintenance of wakefulness, but not in homeostatic regulation of sleep, in mice. J Neurosci 30:4382-4389.

Sakai K and Crochet S (2001) Differentiation of presumed serotonergic dorsal raphe neurons in relation to behavior and wake-sleep states. Neuroscience 104: 1141-1155.

Sos KE, Mayer MI, Cserép C, Takács FS, Szőnyi A, Freund TF, and Nyiri G (2016) Cellular architecture and transmitter phenotypes of neurons of the mouse median raphe region. Brain Struct Funct 222:287-299.

Tanaka M, Nagashima K, McAllen RM, and Kanosue K (2002) Role of the medullary raphé in thermoregulatory vasomotor control in rats. $J$ Physiol 540: $657-664$.

Trulson ME and Jacobs BL (1979) Raphe unit activity in freely moving cats: correlation with level of behavioral arousal. Brain Res 163:135-150.

Whitney MS, Shemery AM, Yaw AM, Donovan LJ, Glass JD, and Deneris ES (2016) Adult brain serotonin deficiency causes hyperactivity, circadian disruption, and elimination of siestas. $J$ Neurosci 36:9828-9842.

Zhao ZQ, Scott M, Chiechio S, Wang JS, Renner KJ, Gereau RW, IV, Johnson RL, Deneris ES, and Chen ZF (2006) Lmx1b is required for maintenance of central serotonergic neurons and mice lacking central serotonergic system exhibit normal locomotor activity. J Neurosci 26:12781-12788.

Address correspondence to: Dr. Yi Rao, Peking University, 5 Yiheyuan Rd., Beijing 100871, China. E-mail: yrao@pku.edu.cn 